Author affiliations appear at the end of this article.

Submitted May 7, 2010; accepted December 20, 2011; published online ahead of print at www.jco.org on February 27, 2012

Supported in part by Grants No. HL072705, HL078522, HL053392, CA127642, CA068484, HD052104, Al50274, CA068484, HD052102, HL087708, HL079233, HL004537, HL087000, HL007188, HL094100, HL095127, and HD80002 from the National Institutes of Health, and by the Michael Garil Fund, the Children's Cardiomyopathy Foundation, the University of Miami Women's Cancer Association, the Lance Armstrong Foundation, Roche Diagnostics, Pfizer, and Novartis.

Presented at the Fifth proCardio Symposium, Seville, Spain, May 11-12, 2007; American Heart Association Scientific Sessions, Orlando, FL, November 3-7, 2007; and American Heart Association Scientific Sessions Chicago, IL, November 13-17, 2010.

Authors' disclosures of potential conflicts of interest and author contributions are found at the end of this article.

Clinical Trials repository link available on JCO.org.

Corresponding author: Steven E. Lipshultz, MD, Department of Pediatrics (D820), University of Miami Miller School of Medicine, PO Box 016820 Miami, FL 33101; e-mail: slipshultz@ med.miami.edu.

(C) 2012 by American Society of Clinical Oncology

0732-183X/12/3010-1042/\$20.00

DOI: $10.1200 / J C O .2010 .30 .3404$

\title{
Changes in Cardiac Biomarkers During Doxorubicin Treatment of Pediatric Patients With High-Risk Acute Lymphoblastic Leukemia: Associations With Long-Term Echocardiographic Outcomes
}

Steven E. Lipshultz, Tracie L. Miller, Rebecca E. Scully, Stuart R. Lipsitz, Nader Rifai, Lewis B. Silverman, Steven D. Colan, Donna S. Neuberg, Suzanne E. Dahlberg, Jacqueline M. Henkel, Barbara L. Asselin, Uma H. Athale, Luis A. Clavell, Caroline Laverdière, Bruno Michon, Marshall A. Schorin, and Stephen E. Sallan

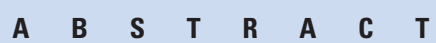

\section{Purpose}

Doxorubicin causes cardiac injury and cardiomyopathy in children with acute lymphoblastic leukemia (ALL). Measuring biomarkers during therapy might help individualize treatment by immediately identifying cardiac injury and cardiomyopathy.

\section{Patients and Methods}

Children with high-risk ALL were randomly assigned to receive doxorubicin alone ( $\mathrm{n}=100 ; 75$ analyzed) or doxorubicin with dexrazoxane ( $n=105 ; 81$ analyzed). Echocardiograms and serial serum measurements of cardiac troponin T (cTnT; cardiac injury biomarker), N-terminal pro-brain natriuretic peptide (NT-proBNP; cardiomyopathy biomarker), and high-sensitivity C-reactive protein (hsCRP; inflammatory biomarker) were obtained before, during, and after treatment.

\section{Results}

cTnT levels were increased in $12 \%$ of children in the doxorubicin group and in $13 \%$ of the doxorubicin-dexrazoxane group before treatment but in $47 \%$ and $13 \%$, respectively, after treatment $(P=.005)$. NT-proBNP levels were increased in $89 \%$ of children in the doxorubicin group and in $92 \%$ of children in the doxorubicin-dexrazoxane group before treatment but in only $48 \%$ and $20 \%$, respectively, after treatment $(P=.07)$. The percentage of children with increased hsCRP levels did not differ between groups at any time. In the first 90 days of treatment, detectable increases in cTnT were associated with abnormally reduced left ventricular (LV) mass and LV end-diastolic posterior wall thickness 4 years later $(P<.01)$; increases in NT-proBNP were related to an abnormal LV thickness-to-dimension ratio, suggesting LV remodeling, 4 years later $(P=.01)$. Increases in hsCRP were not associated with any echocardiographic variables.

\section{Conclusion}

cTnT and NT-proBNP may hold promise as biomarkers of cardiotoxicity in children with high-risk ALL. Definitive validation studies are required to fully establish their range of clinical utility.

\section{J Clin Oncol 30:1042-1049. (C) 2012 by American Society of Clinical Oncology}

\section{INTRODUCTION}

Doxorubicin treats hematologic malignancies and solid tumors ${ }^{1}$ but is limited by cardiotoxicity. ${ }^{2-6}$ Doxorubicin cardiotoxicity is dose limiting during treatment and causes long-term morbidity and early mortality among cancer survivors. ${ }^{4-6}$ Because doxorubicin-induced cardiac damage is progressive and often irreversible, minimizing cardiac injury during treatment to prevent late cardiomyopathy is critical. ${ }^{7-10}$ For example, the concomitant administration of dexrazoxane substantially reduces cardiotoxicity. ${ }^{9-11}$

Identifying risk factors has helped characterize groups at high risk for cardiac injury, ${ }^{2-6}$ but tolerance of chemotherapy and predisposition to cardiac damage vary substantially among patients. Although cardiac imaging techniques can assess heart structure and function during and after therapy, they lack the sensitivity to detect early treatment-induced cardiac injury and the specificity to distinguish myocardial injury that may have late 
implications in long-term survivors from short-term changes in left ventricular (LV) loading conditions or transient cytokinemediated myocardial depressant effects. ${ }^{8}$ Serial endomyocardial biopsies are impractical. ${ }^{12}$

Serial monitoring of circulating biomarker levels can evaluate cardiotoxicity during and after therapy accurately and efficiently. ${ }^{13-16}$ Increases in cardiac troponin $\mathrm{T}$ (cTnT) and cardiac troponin I (cTnI) reflect cardiac damage and often indicate irreversible cardiomyocyte necrosis. ${ }^{17-21}$ Chronic increases in N-terminal probrain natriuretic peptide (NT-proBNP) indicate increased LV wall stress associated with pressure and volume overload and increased diastolic pressure. ${ }^{22-33}$

As a global inflammatory marker, high-sensitivity C-reactive protein (hsCRP) is used to assess patient health. ${ }^{34}$ Increases in serum hsCRP may indicate cardiac stress because inflammation is important in heart disease. ${ }^{35,36}$

Biomarkers may improve cardiotoxicity monitoring during and after doxorubicin treatment. ${ }^{5,6,9,13-16,18,19}$ Thus, in this study, we characterized trends in cTnT, NT-proBNP, and hsCRP levels in children with high-risk acute lymphoblastic leukemia (ALL) before, during, and after treatment with doxorubicin to examine the effect of doxorubicin with or without dexrazoxane on biomarker levels and to identify the relationships between these biomarkers and echocardiographic variables over time.

\section{PATIENTS AND METHODS}

\section{Patients}

This study was part of the Dana-Farber Cancer Institute Childhood ALL Consortium Protocol 95-01, which enrolled patients younger than age 18 years with newly diagnosed ALL between January 1996 and September $2000 .{ }^{10}$ The institutional review board at each participating institution approved the protocol before patient enrollment. Informed consent was obtained from patients, parents, or guardians before starting therapy.

Serial serum samples and echocardiograms were obtained in children with high-risk ALL, that is, those who at diagnosis were younger than 12 months or more than 10 years of age and had any of the following: a leukocyte count of $\geq 50,000$ cells $/ \mu \mathrm{L}$, a T-cell immunophenotype, an anterior mediastinal mass, or CNS disease.

\section{Therapy and Data Collection}

In addition to multiagent chemotherapy and CNS radiation, all children with high-risk ALL received two doses of doxorubicin $\left(30 \mathrm{mg} / \mathrm{m}^{2} /\right.$ dose $)$ during remission induction and then eight more doses $\left(30 \mathrm{mg} / \mathrm{m}^{2} /\right.$ dose $)$ every 3 weeks during postremission intensification to a cumulative dose of $300 \mathrm{mg} /$ $\mathrm{m}^{2} \cdot{ }^{10}$ No doxorubicin was given after about 9 months of therapy. In addition, patients were randomly assigned to receive doxorubicin alone or doxorubicin immediately preceded by dexrazoxane $\left(300 \mathrm{mg} / \mathrm{m}^{2} /\right.$ dose $)$.

Serum for biomarker assays was collected at diagnosis (before doxorubicin therapy), daily after induction doses of doxorubicin for days 1 through 7,7 days after a doxorubicin dose during the consolidation phase, and at the end of doxorubicin therapy. Serum samples were sent to a central laboratory, frozen immediately, and stored at $-70^{\circ} \mathrm{C}$ until assayed. Hemolyzed samples or samples with insufficient material could not be assayed and were excluded. Investigators performing serum assays and echocardiographic evaluations remained blinded throughout the study. An independent data safety monitoring committee reviewed accrual, toxicity, and troponin results every 6 months and released the troponin results for publication after all children had completed doxorubicin treatment. ${ }^{9}$

\section{Biomarker Assays}

Levels of hsCRP were determined by using the Tina-quant C-reactive protein [latex] high-sensitive assay (Roche Diagnostics, Indianapolis, IN; sen- sitivity, $0.03 \mathrm{mg} / \mathrm{L}$ ). Levels of NT-proBNP were measured by using the Elecsys immunoanalyzer (Roche Diagnostics; sensitivity, $5 \mathrm{pg} / \mathrm{mL}$ ). Levels of cTnT were measured by using the Elecsys Troponin-T STAT Immunoassay (Roche Diagnostics; sensitivity, $0.01 \mathrm{ng} / \mathrm{mL}) .{ }^{18} \mathrm{CTnI}$ levels were measured on $40 \mathrm{sam}$ ples from 18 children selected by cTnT level and available volume; 20 samples with increased cTnT levels and 20 without were analyzed for cTnI levels by using the Singulex ERENNA System (Singulex, Alameda, CA; sensitivity, $0.0002 \mathrm{ng} / \mathrm{mL}$ ). ${ }^{37}$ Abnormal values were defined as follows: for cTnT and cTnI, any detectable amount; for NT-proBNP, levels $\geq 150 \mathrm{pg} / \mathrm{mL}$ in infants younger than 1 year or $\geq 100 \mathrm{pg} / \mathrm{mL}$ in children age 1 year or older; and for hsCRP, levels $\geq 1.9 \mathrm{mg} / \mathrm{L}$.

\section{Echocardiographic Measurements}

Echocardiograms were obtained at diagnosis, after doxorubicin therapy, and every 2 years thereafter. LV status was assessed with systolic and diastolic dimensions; mass; end-diastolic posterior wall thickness; thickness-todimension ratio; and fractional shortening, an index of LV systolic performance influenced by heart rate, preload, afterload, and contractility. ${ }^{9-11}$ Echocardiograms were obtained at local treatment sites and remeasured at a single facility by blinded study staff. ${ }^{9}$ Children were eligible for cardiac follow-up throughout their first continuous complete remission.

\section{Statistical Methods}

Means of normally distributed outcome variables (eg, echocardiographic measurements) were compared with $t$ tests; means of non-normally distributed variables (eg, age) were compared with Wilcoxon rank sum tests. Differences in proportions of dichotomous values (eg, abnormal cTnT) between groups were compared with Fisher's exact tests.

To adjust for growth-related changes, echocardiographic data were standardized by age ( $L V$ fractional shortening and blood pressure) or body surface area (LV mass, LV dimension, and LV wall thickness). A Z score was then calculated from normal predicted values from a regression model by using values from 285 normal children ${ }^{38}$ in whom body surface area ranged from 0.2 to $2.2 \mathrm{~m}^{2}$, and who had normal height, weight, height-for-weight percentiles, and blood pressure and no evidence of cardiac or other disorders.

We did not assume that all follow-up biomarker and echocardiographic data would be collected from all patients at predetermined time points. Thus, although we collected blood samples from as many patients as possible until 2 months after doxorubicin treatment, we could not calculate the proportion of patients with abnormal values at each time point (eg, monthly).

Each time a patient was observed, we created a binary outcome for each biomarker (abnormal or normal). The probability of an abnormal outcome was then estimated from logistic regression models as a linear, quadratic, and cubic function of the time of measurement by using all data from all time points on all patients. Then we estimated the probability of an abnormal value at each month for 9 months from this logistic regression model (using months 0 through 9 in the model).

Given repeated measures on the same patient, we fit a logistic regression model for the probability of abnormal outcomes occurring over time by repeated measures (generalized estimating equations) logistic regression models in SAS PROC GLIMMIX (SAS Institute, Cary, NC) that modeled the probability of an abnormal outcome as a linear, quadratic, and cubic function of time. (See Appendix [online only] for more details on the modeling methods.) We modeled the correlation between outcomes measured at a pair of times on the same child with an autoregressive structure. We plotted the probability of abnormal cardiac biomarkers from this logistic regression against time to estimate the probability of an abnormal biomarker level at a given time point. A similar mixed model was used to model echocardiographic $\mathrm{Z}$ scores after doxorubicin treatment as a function of time, treatment, and increased abnormal cardiac biomarkers during therapy (dichotomized as abnormal or normal). From this mixed model, we estimated the mean echocardiographic $\mathrm{Z}$ scores at 4 years as a function of abnormal cardiac biomarker levels during therapy. We calculated $95 \%$ CIs for the predicted probabilities by transforming a 95\% CI for the logit at each time point (calculated as the logit \pm 1.96 times the SE of the logit).

Although the number of dropouts did not differ between groups, our approach protects against biases caused by patients on one treatment being 


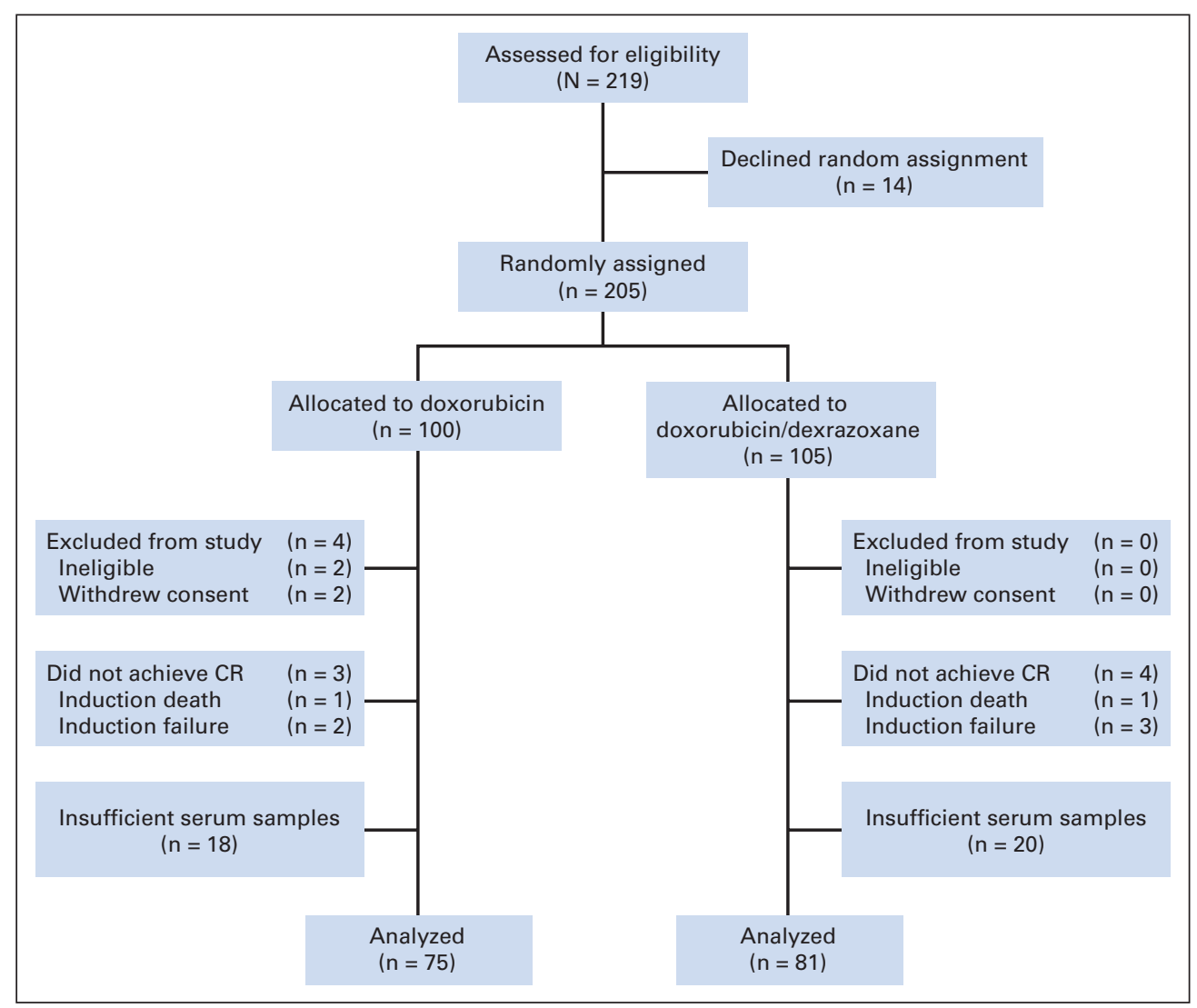

Fig 1. CONSORT diagram shows allocation and disposition of patients with highrisk acute lymphoblastic leukemia in the Dana-Farber Cancer Institute Childhood Acute Lymphoblastic Leukemia Consortium Protocol 95-01 randomized controlled trial. $\mathrm{CR}$, complete remission.

seen more often and followed for longer periods than patients on the other treatment, as might occur in patients with poorer heart function on that treatment. The logistic regression model formulation in SAS PROC GLIMMIX adjusts for this type of missing follow-up data ${ }^{38-40}$ and ensures that data from patients with poor cardiac function who came back most often for repeat testing were not weighted excessively and did not disproportionately influence the tails of the plots.

\section{RESULTS}

\section{Patient Characteristics}

Between 1996 and 2000, 491 children were enrolled on Protocol 95-01, of whom 219 were high risk. Of these, 14 declined assignment. Of the remaining 205, 100 (49\%) were randomly assigned to receive doxorubicin alone and 105 (51\%) to receive doxorubicin with dexrazoxane. Of the 205 patients, 75 (75\%) in the doxorubicin group and 81 $(77 \%)$ in the doxorubicin-dexrazoxane group had evaluable serum samples, providing a sample size of 156 patients (Fig 1).

In children with any serum samples evaluated during treatment, the percentage of girls was similar in both groups. The median cumulative dose of doxorubicin was $300 \mathrm{mg} / \mathrm{m}^{2}$ for both groups. The percentage of children contributing evaluable samples did not differ between groups on any characteristic (data not shown). The mean number of samples per child was also similar between groups (about 15) and among specific biomarkers by study intervals (Table 1). Patients with biomarkers measured before doxorubicin therapy were similar between groups $(P>.3)$ on all observed baseline characteristics.

\section{cTnT}

At baseline, cTnT was detected in approximately $12 \%$ of children in both groups (Table 2). During treatment, the cumulative percentage of children with detectable cTnT levels increased to $47 \%$ (35 of 75 ) in the doxorubicin group and to $20 \%$ (16 of 80 ) in the doxorubicin-dexrazoxane group $(P<.001$; Table 2$)$. After therapy ended, detectable levels were found in $47 \%$ (14 of 30 ) of children in the doxorubicin group but only in $13 \%$ (four of 32 ) of the doxorubicin-dexrazoxane group $(P=.005$; Table 2$)$. Similarly, the percentage of children with multiple detections at any study interval was also higher in the doxorubicin group (40\% [30 of 75] $v 13 \%$ [11 of 81 ]; $P<.001$; Table 2 ).

A repeated measure model revealed that there were no significant treatment differences at baseline, but then 1.8 months after assignment, the percentage of samples with detectable cTnT levels in the doxorubicin group became significantly higher than that in the doxorubicin-dexrazoxane group and remained so until the end of treatment (Fig 2A). Further, overall, the repeated measures model found significant differences in $\mathrm{CTnT}(P<.001)$ between the groups in Figure 2A.

\section{NT-proBNP}

At baseline, the percentages of children with increased NTproBNP levels did not differ between groups (Table 2). During treatment, the percentage of children with increased NT-proBNP levels decreased in the doxorubicin-dexrazoxane group but increased in the doxorubicin group $(P=.005$; Table 2$)$. After therapy, the 


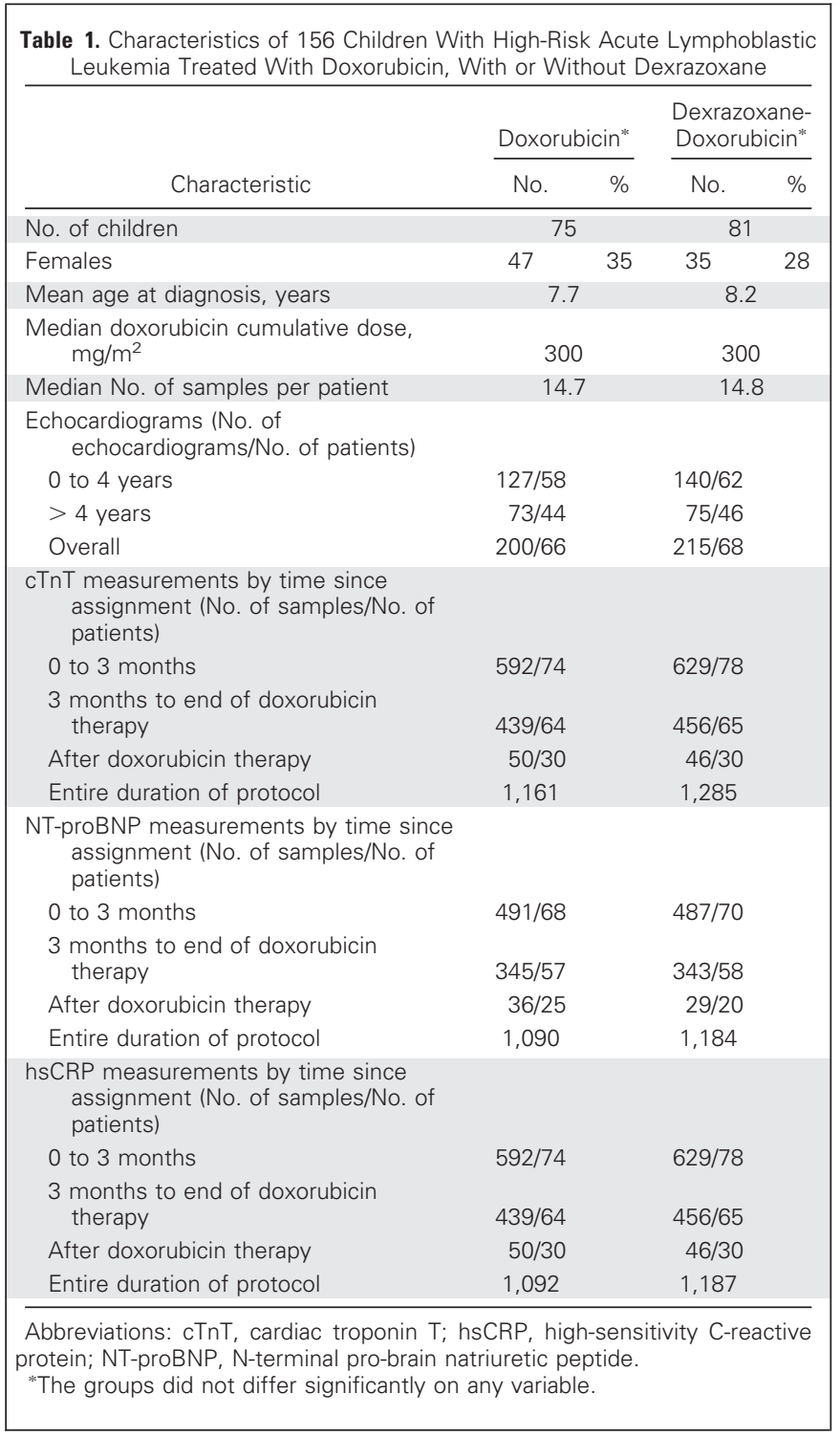

percentage decreased in both groups but was lower in the doxorubicindexrazoxane group (48\% [12 of 25] $v 20 \%$ [four of 20]; $P=.07$; Table $2)$. The percentage of children with any increase in NT-proBNP was also significantly lower in the doxorubicin-dexrazoxane group (86\% [63 of 73] $v 100 \%$ [70 of 70]; $P=.001$; Table 2). The doxorubicin group also had a significantly higher percentage of children with multiple NT-proBNP increases $(P=.007$, Table 2$)$.

In the repeated measure model, the percentage of samples with increased NT-proBNP levels was similar between the groups at baseline (Fig 2B). During treatment, the percentage fell to 35\% (from 83\%) in the doxorubicin group and to $16 \%$ (from $87 \%$ ) in the doxorubicindexrazoxane group. After the end of treatment, the percentage of samples with increases rose in the doxorubicin group but continued to decrease in the doxorubicin-dexrazoxane group. The percentage of samples with increased NT-proBNP was significantly lower in the doxorubicin-dexrazoxane group during treatment $(P<.001$; Fig $2 \mathrm{~B})$. Further, overall, the repeated measures model found significant differences in NT-proBNP $(P<.001)$ between the groups in Figure $2 \mathrm{~B}$.

\begin{tabular}{|c|c|c|c|c|c|}
\hline \multirow[b]{2}{*}{ Biomarker and Study Interval } & \multicolumn{2}{|c|}{ Doxorubicin } & \multicolumn{2}{|c|}{$\begin{array}{l}\text { Dexrazoxane- } \\
\text { Doxorubicin }\end{array}$} & \multirow[b]{2}{*}{$P^{*}$} \\
\hline & $\%$ & $\begin{array}{c}\text { No. of } \\
\text { Patients With } \\
\text { Elevations/No. } \\
\text { of Patients }\end{array}$ & $\%$ & $\begin{array}{c}\text { No. of } \\
\text { Patients With } \\
\text { Elevations/No. } \\
\text { of Patients }\end{array}$ & \\
\hline \multicolumn{6}{|l|}{$\overline{c T n T \dagger}$} \\
\hline Any increase & 50.7 & $38 / 75$ & 27.1 & $22 / 81$ & .003 \\
\hline Before doxorubicin therapy & 11.7 & $6 / 51$ & 12.7 & $7 / 55$ & .99 \\
\hline During doxorubicin therapy & 46.6 & $35 / 75$ & 20.0 & $16 / 80$ & $<.001$ \\
\hline After doxorubicin therapy & 46.6 & $14 / 30$ & 12.5 & $4 / 32$ & .005 \\
\hline Multiple increases & 40.0 & $30 / 75$ & 13.4 & $11 / 81$ & $<.001$ \\
\hline \multicolumn{6}{|l|}{ NT-proBNP $\ddagger$} \\
\hline Any increase & 100 & $70 / 70$ & 86.3 & $63 / 73$ & .001 \\
\hline Before doxorubicin therapy & 88.8 & $32 / 36$ & 91.8 & $34 / 37$ & .71 \\
\hline During doxorubicin therapy & 98.5 & $68 / 69$ & 84.9 & $62 / 73$ & .005 \\
\hline After doxorubicin therapy & 48.0 & $12 / 25$ & 20.0 & $4 / 20$ & .07 \\
\hline Multiple increases & 90.4 & $66 / 73$ & 78.0 & $57 / 73$ & .007 \\
\hline \multicolumn{6}{|l|}{ hsCRP§ } \\
\hline Any increase & 95.7 & $67 / 70$ & 89.0 & $65 / 73$ & .20 \\
\hline Before doxorubicin therapy & 83.3 & $30 / 36$ & 81.0 & $30 / 37$ & .99 \\
\hline During doxorubicin therapy & 95.6 & $66 / 69$ & 87.6 & $64 / 73$ & .13 \\
\hline After doxorubicin therapy & 45.8 & $11 / 24$ & 42.1 & $8 / 19$ & .99 \\
\hline Multiple increases & 88.5 & $62 / 70$ & 76.7 & $56 / 73$ & .08 \\
\hline
\end{tabular}

Abbreviations: cTnT, cardiac troponin T; hsCRP, high-sensitivity C-reactive protein; NT-proBNP, N-terminal pro-brain natriuretic peptide.

*Fisher's exact test.

tIncreased cTnT defined as $>0.01 \mathrm{ng} / \mathrm{mL}$.

fIncreased NT-proBNP defined as $\geq 100 \mathrm{pg} / \mathrm{mL}$ in patients $\geq 1$ year old and $\geq 150 \mathrm{pg} / \mathrm{mL}$ in patients $<1$ year old.

§lncreased hsCRP defined as $\geq 1.9 \mathrm{mg} / \mathrm{L}$.

\section{hsCRP}

The percentage of children with increased hsCRP levels did not differ significantly between groups before, during, or after treatment, nor did the percentage with multiple increases (Table 2 ). In the repeated measure model, the percentage of samples with increased hsCRP levels did not differ significantly between groups at baseline (Fig 2C). During treatment, the percentage fell in both groups, with the doxorubicin group reaching a nadir of 16\% (from 79\%) 3.6 months after starting therapy before increasing toward the end of treatment and becoming significantly greater than that in the doxorubicin-dexrazoxane group (Fig 2C). Further, overall, the repeated measures model found significant differences in hsCRP $(P=.04)$ between the groups in Figure 2C.

\section{cTnI}

During treatment, cTnI levels were measured in a subset of samples. The median cTnI level during the first month of treatment was $1.75 \mathrm{pg} / \mathrm{mL}$ for the doxorubicin group and $4.42 \mathrm{pg} / \mathrm{mL}$ for the doxorubicin-dexrazoxane group $(P=.26)$. By the end of treatment, the median value for the doxorubicin group (41.65 $\mathrm{pg} / \mathrm{mL}$ ) was significantly higher than that for the doxorubicindexrazoxane group $(1.44 \mathrm{pg} / \mathrm{mL} ; P<.001$; Fig 3$)$.

\section{Association of Biomarker Levels That Increased During Treatment With Echocardiographic Findings 4 Years Later}

A total of 415 echocardiograms were obtained from 134 children (200 from 66 children in the doxorubicin group and 215 from 68 


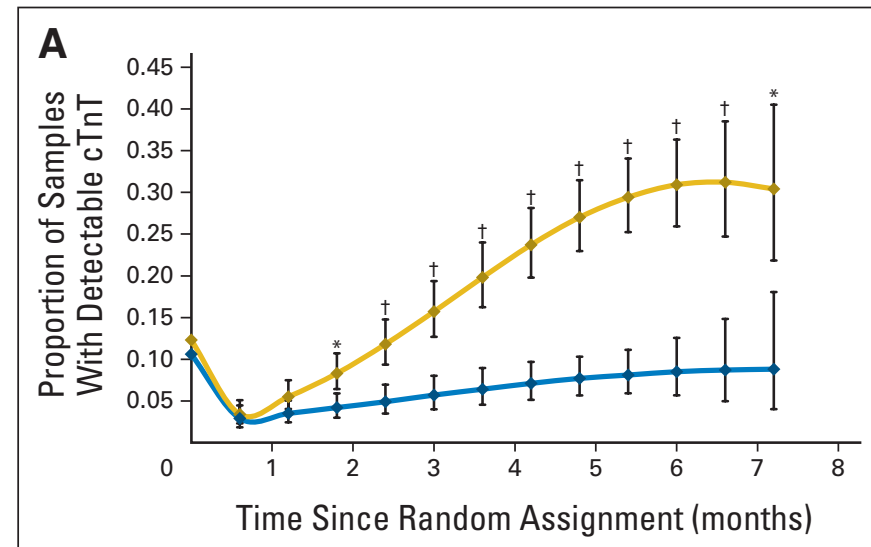

B
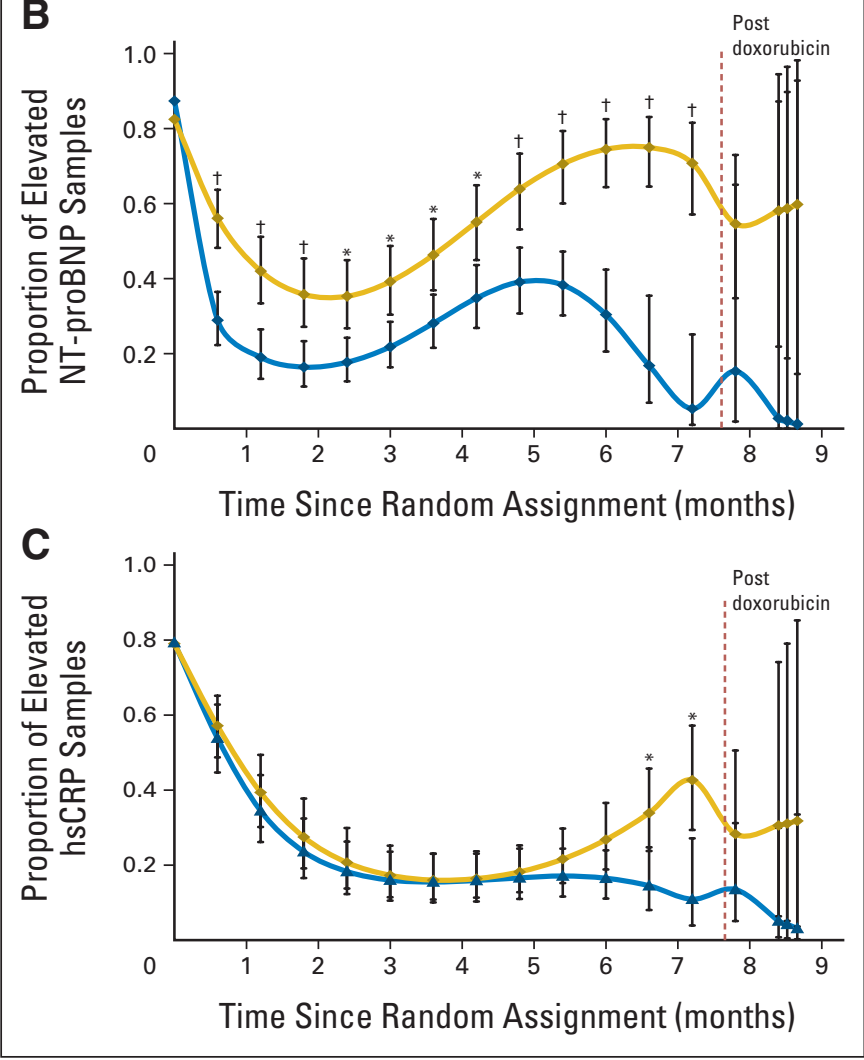

Fig 2. (A) Model-based estimated probability of having an increased cardiac troponin $\mathrm{T}(\mathrm{c} T \mathrm{nT})$ level at each depicted time point in patients treated with doxorubicin, with or without dexrazoxane. The doxorubicin-dexrazoxane group is indicated by the blue line, the doxorubicin group by the gold line. Vertical bars show $95 \% \mathrm{Cls}$. Increased cTnT is defined as a value greater than $0.01 \mathrm{ng} / \mathrm{mL}$. ${ }^{*} P$ value versus dexrazoxane group $\leq .05$; $\dagger P$ value versus dexrazoxane group $\leq$ .001. An overall test for dexrazoxane effect during treatment was significant $(P<$ .001). (B) Model-based estimated probability of having an increased N-terminal pro-brain natriuretic peptide (NT-proBNP) level at each depicted time point in patients treated with doxorubicin with or without dexrazoxane. The dexrazoxane group is indicated by the blue line, the doxorubicin group by the gold line. Vertical bars show $95 \% \mathrm{Cls}$. Increased NT-proBNP is defined as a value $\geq 150 \mathrm{pg} / \mathrm{mL}$ for children younger than 1 year old and a value $\geq 100 \mathrm{pg} / \mathrm{mL}$ for children age $\geq 1$ year. ${ }^{*} P$ value versus dexrazoxane group $\leq .05$; $\dagger P$ value versus dexrazoxane group $\leq .001$. An overall test for dexrazoxane effect during treatment was significant $(P<.001)$ and after treatment was not significant $(P=.24)$. (C) Model-based estimated probability of having an increased high-sensitivity C-reactive protein (hsCRP) level at each depicted time point in patients treated with doxorubicin with or without dexrazoxane. The dexrazoxane group is indicated by the blue line, the doxorubicin group by the gold line. Vertical bars show $95 \% \mathrm{Cls}$. Increased hsCRP is defined as a level $\geq 1.9 \mathrm{mg} / \mathrm{L}$. ${ }^{*} P$ value versus dexrazoxane group $\leq .05$. Overall tests for dexrazoxane effect during treatment $(P=.08)$ and after treatment $(P=.49)$ were not significant.

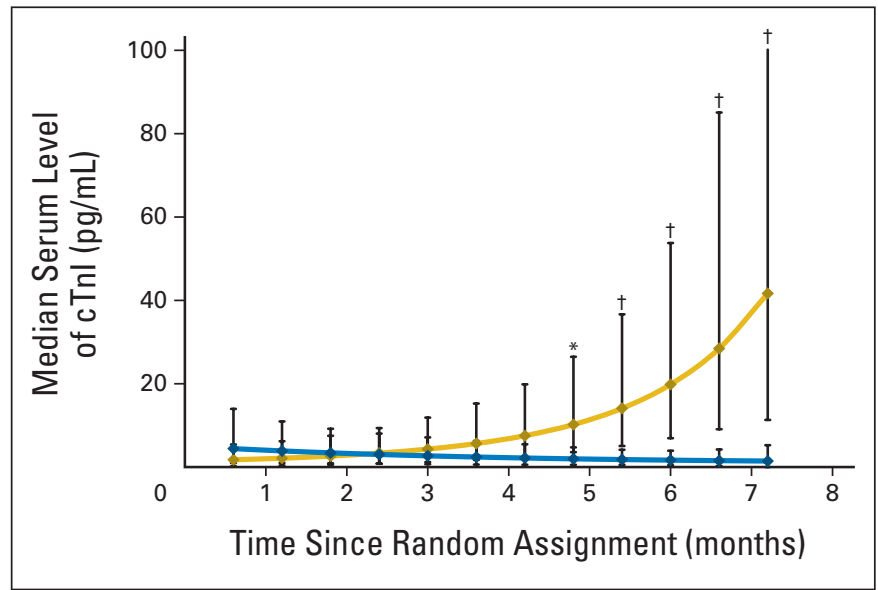

Fig 3. Median serum cardiac troponin I (cTnl) levels during doxorubicin therapy of a 40-sample subset from 18 children with high-risk acute lymphoblastic leukemia. The dexrazoxane group is indicated by the blue line, the doxorubicin group by the gold line. Vertical bars show $95 \% \mathrm{Cls}$. * P value versus dexrazoxanedoxorubicin group $\leq .05 ;+P$ value versus dexrazoxane group $\leq .001$.

children in the doxorubicin-dexrazoxane group). Mean follow-up time was 4.1 years (range, 1.5 to 7.9 years). Increased cTnT levels during the first 90 days of doxorubicin therapy were significantly associated with lower LV mass and LV end-diastolic posterior wall thickness 4 years later; they were marginally significantly associated with lower LV thickness-to-dimension ratios (Table 3). Increases in NT-proBNP detected in the first 90 days of treatment were significantly associated with changes in the LV thickness-to-dimension ratio 4 years later (Table 3 ).

During the last 90 days of treatment, increased levels of cTnT, hsCRP, or NT-proBNP were not significantly related to any echocardiographic outcome 4 years later (Table 4 ).

\section{DISCUSSION}

Our results suggest the utility of assessing cardiac status with biomarkers such as cTnT and NT-proBNP in children receiving doxorubicin for ALL. Serum cTnT levels increased during the first 90 days of therapy, indicating cardiomyocyte damage or death; they were significantly associated with reduced LV mass and LV end-diastolic posterior wall thickness and were marginally associated with a reduced LV thickness-to-dimension ratio 4 years later. Similarly, abnormal NTproBNP levels during the first 90 days of therapy were associated with an abnormal LV thickness-to-dimension ratio, suggesting pathologic LV remodeling 4 years later. In addition, before, during, and after treatment, a higher percentage of children had increased levels of NT-proBNP (indicating increased LV wall stress) than had abnormal cTnT levels (indicating cardiomyocyte death), suggesting that NTproBNP may detect cardiac stress before irreversible cardiomyocyte death.

There are some well-defined trends in biomarkers throughout doxorubicin treatment. Immediately after doxorubicin treatment began, the proportion of children with increased levels of NT-proBNP, hsCRP, and cTnT decreased substantially. Many children with highrisk ALL are quite ill at presentation. With supportive care and intensive multidrug induction chemotherapy, these children often 
Table 3. Associations Between Biomarkers Measured in the First 90 Days of Doxorubicin Treatment and Echocardiographic Outcomes 4 Years Later in 156 Doxorubicin-Treated Children With High-Risk Acute Lymphoblastic Leukemia

\begin{tabular}{|c|c|c|c|c|}
\hline Left Ventricular Characteristic & $\begin{array}{c}\text { Mean Z } \\
\text { Score }\end{array}$ & SE & $P \vee 0$ & $\begin{array}{r}P \text { for } \\
\text { Equal } \\
\text { Means }\end{array}$ \\
\hline \multicolumn{5}{|l|}{ Fractional shortening } \\
\hline Increased cTnT* & -0.84 & 0.26 & .001 & \\
\hline Normal cTnT & -0.91 & 0.20 & $<.001$ & .82 \\
\hline \multicolumn{5}{|l|}{ Mass } \\
\hline Increased cTnT & -1.36 & 0.19 & $<.001$ & \\
\hline Normal cTnT & -0.68 & 0.14 & $<.001$ & .004 \\
\hline \multicolumn{5}{|l|}{ End-diastolic dimension } \\
\hline Increased cTnT & -0.34 & 0.25 & .17 & \\
\hline Normal cTnT & -0.06 & 0.17 & .74 & .35 \\
\hline \multicolumn{5}{|l|}{ End-systolic dimension } \\
\hline Increased cTnT & 0.13 & 0.26 & .60 & \\
\hline Normal cTnT & 0.37 & 0.18 & .04 & .46 \\
\hline \multicolumn{5}{|l|}{ End-diastolic posterior wall thickness } \\
\hline Increased cTnT & -1.51 & 0.24 & $<.001$ & \\
\hline Normal cTnT & -0.74 & 0.17 & $<.001$ & .008 \\
\hline \multicolumn{5}{|l|}{ Thickness-to-dimension ratio } \\
\hline Increased cTnT & -1.14 & 0.26 & $<.001$ & \\
\hline Normal cTnT & -0.52 & 0.18 & $<.001$ & .054 \\
\hline \multicolumn{5}{|l|}{ Fractional shortening } \\
\hline Increased NT-proBNPt & -0.70 & 0.17 & $<.001$ & \\
\hline Normal NT-proBNP & -1.43 & 0.55 & .01 & .19 \\
\hline \multicolumn{5}{|l|}{ Mass } \\
\hline Increased NT-proBNP & -0.87 & 0.12 & $<.001$ & \\
\hline Normal NT-proBNP & -0.37 & 0.49 & .44 & .33 \\
\hline \multicolumn{5}{|l|}{ End-diastolic dimension } \\
\hline Increased NT-proBNP & 0.02 & 0.12 & .85 & \\
\hline Normal NT-proBNP & -0.69 & 0.65 & .29 & .27 \\
\hline \multicolumn{5}{|l|}{ End-systolic dimension } \\
\hline Increased NT-proBNP & 0.37 & 0.13 & .006 & \\
\hline Normal NT-proBNP & 0.02 & 0.63 & .98 & .58 \\
\hline \multicolumn{5}{|l|}{ End-diastolic posterior wall thickness } \\
\hline Increased NT-proBNP & -1.02 & 0.15 & $<.001$ & \\
\hline Normal NT-proBNP & -0.20 & 0.49 & .68 & .10 \\
\hline \multicolumn{5}{|l|}{ Thickness-to-dimension ratio } \\
\hline Increased NT-proBNP & -0.87 & 0.16 & $<.001$ & \\
\hline Normal NT-proBNP & 0.32 & 0.44 & .47 & .01 \\
\hline \multicolumn{5}{|l|}{ Fractional shortening } \\
\hline Increased hsCRP $\ddagger$ & -0.82 & 0.18 & $<.001$ & \\
\hline Normal hsCRP & -0.60 & 0.47 & .20 & .67 \\
\hline \multicolumn{5}{|l|}{ Mass } \\
\hline Increased hsCRP & -0.85 & 0.12 & $<.001$ & \\
\hline Normal hsCRP & -0.39 & 0.60 & .52 & .47 \\
\hline \multicolumn{5}{|l|}{ End-diastolic dimension } \\
\hline Increased hsCRP & -0.06 & 0.13 & .68 & \\
\hline Normal hsCRP & -0.24 & 0.70 & .73 & .79 \\
\hline \multicolumn{5}{|l|}{ End-systolic dimension } \\
\hline Increased hsCRP & 0.35 & 0.15 & .019 & \\
\hline Normal hsCRP & 0.05 & 0.62 & .93 & .64 \\
\hline \multicolumn{5}{|l|}{ End-diastolic posterior wall thickness } \\
\hline Increased hsCRP & -0.95 & 0.16 & $<.001$ & \\
\hline Normal hsCRP & -0.57 & 0.51 & .27 & .48 \\
\hline \multicolumn{5}{|l|}{ Thickness-to-dimension ratio } \\
\hline Increased hsCRP & -0.78 & 0.16 & $<.001$ & \\
\hline Normal hsCRP & -0.16 & 0.58 & .78 & .23 \\
\hline
\end{tabular}

Abbreviations: cTnT, cardiac troponin $\mathrm{T}$; hsCRP, high-sensitivity C-reactive protein; NT-proBNP, N-terminal pro-brain natriuretic peptide.

${ }^{*}$ Increased cTnT defined as $>0.01 \mathrm{ng} / \mathrm{mL}$.

tIncreased NT-proBNP defined as $\geq 100 \mathrm{pg} / \mathrm{mL}$ in patients $\geq 1$ year old and $\geq 150 \mathrm{pg} / \mathrm{mL}$ in patients $<1$ year old.

$\neq$ Increased hsCRP defined as $\geq 1.9 \mathrm{mg} / \mathrm{L}$.
Table 4. Associations Between Increased Biomarkers Measured in the Last 90 Days of Doxorubicin Treatment and Echocardiographic Outcomes 4 Years Later in 156 Doxorubicin-Treated Children With High-Risk Acute Lymphoblastic Leukemia

\begin{tabular}{|c|c|c|c|c|}
\hline Left Ventricular Characteristic & $\begin{array}{c}\text { Mean Z } \\
\text { Score }\end{array}$ & SE & $P \vee 0$ & $\begin{array}{r}P \text { for } \\
\text { Equal } \\
\text { Means }\end{array}$ \\
\hline \multicolumn{5}{|l|}{ Fractional shortening } \\
\hline Increased cTnT* & -1.04 & 0.33 & .001 & \\
\hline Normal cTnT & -0.81 & 0.24 & .001 & .59 \\
\hline \multicolumn{5}{|l|}{ Mass } \\
\hline Increased cTnT & -1.02 & 0.21 & $<.001$ & \\
\hline Normal cTnT & -0.81 & 0.17 & $<.001$ & .45 \\
\hline \multicolumn{5}{|l|}{ End-diastolic dimension } \\
\hline Increased cTnT & 0.06 & 0.18 & .73 & \\
\hline Normal cTnT & 0.06 & 0.20 & .78 & .67 \\
\hline \multicolumn{5}{|l|}{ End-systolic dimension } \\
\hline Increased cTnT & 0.54 & 0.24 & .02 & \\
\hline Normal cTnT & 0.33 & 0.20 & .10 & .50 \\
\hline \multicolumn{5}{|l|}{ End-diastolic posterior wall thickness } \\
\hline Increased cTnT & -1.12 & 0.26 & $<.001$ & \\
\hline Normal cTnT & -0.86 & 0.18 & $<.001$ & .38 \\
\hline \multicolumn{5}{|l|}{ Thickness-to-dimension ratio } \\
\hline Increased cTnT & -1.02 & 0.27 & $<.001$ & \\
\hline Normal cTnT & -0.61 & 0.20 & .002 & .20 \\
\hline \multicolumn{5}{|l|}{ Fractional shortening } \\
\hline Increased NT-proBNPt & -0.97 & 0.23 & $<.001$ & \\
\hline Normal NT-proBNP & -0.66 & 0.30 & .033 & .40 \\
\hline \multicolumn{5}{|l|}{ Mass } \\
\hline Increased NT-proBNP & -0.93 & 0.14 & $<.001$ & \\
\hline Normal NT-proBNP & -0.53 & 0.37 & .15 & .32 \\
\hline \multicolumn{5}{|l|}{ End-diastolic dimension } \\
\hline Increased NT-proBNP & 0.01 & 0.16 & .97 & \\
\hline Normal NT-proBNP & -0.46 & 0.30 & .13 & .14 \\
\hline \multicolumn{5}{|l|}{ End-systolic dimension } \\
\hline Increased NT-proBNP & 0.46 & 0.18 & .01 & \\
\hline Normal NT-proBNP & -0.03 & 0.32 & .93 & .16 \\
\hline \multicolumn{5}{|l|}{ End-diastolic posterior wall thickness } \\
\hline Increased NT-proBNP & -0.92 & 0.17 & $<.001$ & \\
\hline Normal NT-proBNP & -0.68 & 0.32 & .04 & .51 \\
\hline \multicolumn{5}{|l|}{ Thickness-to-dimension ratio } \\
\hline Increased NT-proBNP & -0.80 & 0.18 & $<.001$ & \\
\hline Normal NT-proBNP & -0.09 & 0.37 & .80 & .09 \\
\hline \multicolumn{5}{|l|}{ Fractional shortening } \\
\hline Increased hsCRP§ & -0.94 & 0.24 & $<.001$ & \\
\hline Normal hsCRP & -0.96 & 0.31 & .002 & .95 \\
\hline \multicolumn{5}{|l|}{ Mass } \\
\hline Increased hsCRP & -0.79 & 0.24 & .001 & \\
\hline Normal hsCRP & -0.88 & 0.18 & $<.001$ & .77 \\
\hline \multicolumn{5}{|l|}{ End-diastolic dimension } \\
\hline Increased hsCRP & 0.21 & 0.19 & .28 & \\
\hline Normal hsCRP & -0.31 & 0.21 & .14 & .06 \\
\hline \multicolumn{5}{|l|}{ End-systolic dimension } \\
\hline Increased hsCRP & 0.61 & 0.20 & .003 & \\
\hline Normal hsCRP & 0.21 & 0.25 & .40 & .21 \\
\hline \multicolumn{5}{|l|}{ End-diastolic posterior wall thickness } \\
\hline Increased hsCRP & -0.95 & 0.21 & $<.001$ & \\
\hline Normal hsCRP & -0.75 & 0.21 & $<.001$ & .50 \\
\hline \multicolumn{5}{|l|}{ Thickness-to-dimension ratio } \\
\hline Increased hsCRP & -0.92 & 0.21 & $<.001$ & \\
\hline Normal hsCRP & -0.41 & 0.23 & .08 & .11 \\
\hline
\end{tabular}

Abbreviations: cTnT, cardiac troponin $\mathrm{T}$; hsCRP, high-sensitivity C-reactive protein; NT-proBNP, N-terminal pro-brain natriuretic peptide.

${ }^{*}$ Increased cTnT defined as $>0.01 \mathrm{ng} / \mathrm{mL}$.

tIncreased NT-proBNP defined as $\geq 100 \mathrm{pg} / \mathrm{mL}$ in patients $\geq 1$ year old and $\geq 150 \mathrm{pg} / \mathrm{mL}$ in patients $<1$ year old.

$\S$ Increased hsCRP defined as $\geq 1.9 \mathrm{mg} / \mathrm{L}$. 
markedly improved, consistent with rapidly improved NT-proBNP levels. However, after 2 months, the proportion of children in the doxorubicin-only group with abnormal cTnT and NT-proBNP levels began to rise. This pronounced increase after doxorubicin therapy, which exceeded pretreatment levels by month 3, indicates cardiomyocyte death. ${ }^{9,41}$ Furthermore, the percentage of children with detectable cTnT levels continued to rise, even after doxorubicin treatment had ended, illustrating the persistent, progressive nature of doxorubicin-related cardiac damage.

Among children who received concomitant dexrazoxane, the percentage of samples with detectable cTnT remained stable. Further, throughout treatment, the percentage of samples with increased NTproBNP was consistently lower in the dexrazoxane-treated group. The ability of dexrazoxane to chelate iron and thus lower oxygen-radicalproducing doxorubicin-iron complexes explains not only the reduction in cardiomyocyte death, indicated by reduced cTnT levels, but also the reduction in ventricular stress and inflammation, indicated by decreased NT-proBNP and hsCRP levels.

In contrast, the percentage of samples with increased hsCRP levels in the doxorubicin-only group did not increase until 6 months after starting treatment. By the end of treatment, this percentage was significantly greater than that in the dexrazoxanetreated group. Although hsCRP in adults predicts clinical cardiac events, its use in children deserves attention because increased hsCRP levels are likely associated with worse cardiac characteristics in children with heart failure. ${ }^{42}$

We also measured cTnI levels in 40 samples, 20 with and 20 without increased cTnT levels. Median cTnI levels did not differ significantly between groups until 5 months after assignment, when levels in the doxorubicin-only group progressively increased, becoming significantly higher than those in the dexrazoxane-treated group.

Circulating cardiomyocyte proteins, such as cTnI, have been related to LV dysfunction and to increased cardiovascular risk in adults receiving high-dose chemotherapy. ${ }^{19,21}$ Adults with increased cTnI levels at the start of chemotherapy have an increased risk of cardiac events, and this risk is greater among those with increases persisting until the end of treatment. ${ }^{19}$ In this pilot analysis, increased cTnI levels were strongly related to doxorubicin therapy without dexrazoxane.

Our study found associations between cardiac biomarkers and echocardiograms 4 years after treatment. NT-proBNP, an indicator of cardiomyopathy, ${ }^{26}$ was increased in a higher percentage of children before, during, and after therapy than was cTnT in both groups. In addition, NT-proBNP may be associated with echocardiographic measurements. ${ }^{42}$ We found that NT-proBNP and cTnT levels that increase in the first 90 days of treatment, when patients are most ill, may predict an abnormal LV thickness-to-dimension ratio consistent with LV remodeling years later, suggesting a time when their hearts may be more vulnerable to the cardiotoxic effects of doxorubicin. Our finding of the importance of the first 90 days of cardioprotective therapy suggests that current recommendations to add dexrazoxane later in the course of doxorubicin therapy might require re-evaluation.

Similarly, serum NT-proBNP and cTnT levels were highly associated, suggesting that monitoring NT-proBNP in these children might predict which children are at higher risk for cardiotoxicity, allowing for treatment modification before irreversible cardiomyocyte damage.

This study has some limitations. It was not designed to determine sensitivity or specificity or to establish cutoffs to guide clinical decisions. However, our results should inform clinical trials and prospective studies assessing the trade-off between cancer treatment and cardioprotective efficacy and the associated biomarker-guided clinical decisions.

In conclusion, these results suggest that monitoring NTproBNP and cTnT in children receiving doxorubicin helps assess later echocardiographic status. The results underscore the importance of dexrazoxane in reducing anthracycline cardiotoxicity. A comprehensive panel of biomarkers to assess cardiac status in these patients should be developed.

\section{AUTHORS' DISCLOSURES OF POTENTIAL CONFLICTS OF INTEREST}

The author(s) indicated no potential conflicts of interest.

\section{AUTHOR CONTRIBUTIONS}

Conception and design: Steven E. Lipshultz, Tracie L. Miller, Stuart R. Lipsitz, Lewis B. Silverman, Stephen E. Sallan

Financial support: Steven E. Lipshultz, Stephen E. Sallan

Administrative support: Steven E. Lipshultz, Tracie L. Miller, Rebecca E. Scully, Jacqueline M. Henkel

Provision of study materials or patients: Lewis B. Silverman, Barbara L. Asselin, Uma H. Athale, Luis A. Clavell, Bruno Michon, Marshall A.

Schorin, Stephen E. Sallan

Collection and assembly of data: Steven E. Lipshultz, Tracie L. Miller, Rebecca E. Scully, Nader Rifai, Jacqueline M. Henkel, Uma H. Athale, Luis A. Clavell, Bruno Michon, Marshall A. Schorin

Data analysis and interpretation: Steven E. Lipshultz, Tracie L. Miller, Rebecca E. Scully, Stuart R. Lipsitz, Steven D. Colan, Donna S. Neuberg, Caroline Laverdière, Bruno Michon

Manuscript writing: All authors

Final approval of manuscript: All authors

\section{REFERENCES}

1. Jemal $A$, Siegel $R$, Ward $E$, et al: Cancer statistics, 2008. CA Cancer J Clin 58:71-96, 2008

2. Lipshultz SE, Lipsitz SR, Mone SM, et al: Female sex and drug dose as risk factors for late cardiotoxic effects of doxorubicin therapy for childhood cancer. N Engl J Med 332:1738-1743, 1995

3. Krischer JP, Cuthbertson DD, Epstein S, et al: Risk factors for early anthracycline clinical cardiotoxicity in children: The pediatric oncology group experience. Prog Pediatr Cardiol 8:83-90, 1997

4. Nysom K, Holm K, Lipsitz SR, et al: Relationship between cumulative anthracycline dose and late cardiotoxicity in childhood acute lymphoblastic leukemia. J Clin Oncol 16:545-550, 1998

5. Barry E, Alvarez JA, Scully RE, et al: Anthracycline-induced cardiotoxicty: Course, pathophysiology, prevention and management. Expert Opin Pharmacother 8:1039-1058, 2007

6. Lipshultz SE, Alvarez JA, Scully RE: Anthracycline associated cardiotoxicity in survivors of childhood cancer. Heart 94:525-533, 2008
7. Gianni L, Herman EH, Lipshultz SE, et al: Anthracycline cardiotoxicity: From bench to bedside. J Clin Oncol 26:3777-3784, 2008

8. Ganz WI, Sridhar KS, Ganz SS, et al: Review of tests for monitoring doxorubicin-induced cardiomyopathy. Oncology 53:461-470, 1996

9. Lipshultz SE, Rifai N, Dalton VM, et al: The effect of dexrazoxane on myocardial injury in doxorubicintreated children with acute lymphoblastic leukemia. N Engl J Med 351:145-153, 2004

10. Moghrabi A, Levy DE, Asselin B, et al: Results of the Dana-Farber Cancer Institute ALL Consortium 
Protocol 95-01 for children with acute lymphoblastic leukemia. Blood 109:896-904, 2007

11. Lipshultz SE, Scully RE, Lipsitz SR, et al: Assessment of dexrazoxane as a cardioprotectant in doxorubicin-treated children with high-risk acute lymphoblastic leukemia: Long-term follow-up of a prospective, randomised, multicentre trial. Lancet Oncol 11:950-961, 2010

12. Cooper LT, Baughman KL, Feldman AM, et al: The role of endomyocardial biopsy in the management of cardiovascular disease: A scientific statement from the American Heart Association, the American College of Cardiology, and the European Society of Cardiology, Endorsed by the Heart Failure Society of America and the Heart Failure Association of the European Society of Cardiology. J Am Coll Cardiol 50:1914-1931, 2007

13. Braunwald E: Biomarkers in heart failure. N Engl J Med 358:2148-2159, 2008

14. Horacek JM, Tichy M, Pudil R, et al: Multimarker approach to evaluation of cardiac toxicity during preparative regimen and hematopoietic cell transplantation. Neoplasma 55:532-537, 2008

15. Zethelius B, Berglund L, Sundström J, et al: Use of multiple biomarkers to improve the prediction of death from cardiovascular causes. N Engl J Med 358:2107-2116, 2008

16. Koç $M$, Bozkurt A, Acartürk $E$, et al: Usefulness of $\mathrm{N}$-terminal pro-B-type natriuretic peptide increase with exercise for predicting cardiovascular mortality in patients with heart failure. Am J Cardiol 101:1157-1162, 2008

17. Lipshultz SE, Somers MJ, Lipsitz SR, et al: Serum cardiac troponin and subclinical cardiac status in pediatric chronic renal failure. Pediatrics 112: 79-86, 2003

18. Lipshultz SE, Rifai N, Sallan SE, et al: Predictive value of cardiac troponin $\mathrm{T}$ in pediatric patients at risk for myocardial injury. Circulation 96:26412648, 1997

19. Cardinale D, Sandri MT, Colombo A, et al: Prognostic value of troponin I in cardiac risk stratification of cancer patients undergoing high-dose chemotherapy. Circulation 109:2749-2754, 2004
20. Jaffe AS, Ravkilde J, Roberts R, et al: It's time for a change to a troponin standard. Circulation 102:1216-1220, 2000

21. Cardinale $D$, Sandri MT, Martinoni $A$, et al: Left ventricular dysfunction predicted by early troponin I release after high-dose chemotherapy. J Am Coll Cardiol 36:517-522, 2000

22. Kistorp C, Raymond I, Pedersen F, et al: $\mathrm{N}$-terminal pro-brain natriuretic peptide, C-reactive protein, and urinary albumin levels as predictors of mortality and cardiovascular events in older adults. JAMA 293:1609-1616, 2005

23. Kragelund C, Grønnin B, Køber $L$, et al: $\mathrm{N}$-terminal pro-B-type natriuretic peptide and longterm mortality in stable coronary heart disease. N Engl J Med 352:666-675, 2005

24. Ndrepepa G, Braun S, Niemöller K, et al: Prognostic value of $\mathrm{N}$-terminal pro-brain natriuretic peptide in patients with chronic stable angina. Circulation 112:2102-2107, 2005

25. Omland T, Persson A, Ng L, et al: N-terminal pro-B-type natriuretic peptide and long-term mortality in acute coronary syndromes. Circulation 106: 2913-2918, 2002

26. Bibbins-Domingo $K$, Gupta $R, N a$, et al: $\mathrm{N}$-terminal fragment of the prohormone brain-type natriuretic peptide (NT-proBNP), cardiovascular events, and mortality in patients with stable coronary heart disease. JAMA 297:169-176, 2007

27. Grewal J, McKelvie RS, Persson $\mathrm{H}$, et al: Usefulness of $\mathrm{N}$-terminal pro-brain natriuretic peptide and brain natriuretic peptide to predict cardiovascular outcomes in patients with heart failure and preserved left ventricular ejection fraction. Am J Cardiol 102:733-737, 2008

28. Lorgis L, Zeller M, Dentan G, et al: Prognostic value of $\mathrm{N}$-terminal pro-brain natriuretic peptide in elderly people with acute myocardial infarction: Prospective observation study. BMJ 338:b1605, 2009

29. Masson S, Latini R: Amino-terminal pro-Btype natriuretic peptides and prognosis in chronic heart failure. Am J Cardiol 101:56-60, 2008

30. Dini FL, Conti U, Fontanive $P$, et al: Prognostic value of $\mathrm{N}$-terminal pro-type-b natriuretic peptide and Doppler left ventricular diastolic variables in patients with chronic systolic heart failure stabilized by therapy. Am J Cardiol 102:463-468, 2008

31. Hunt PJ, Yandle TG, Nicholls MG, et al: The amino-terminal portion of pro-brain natriuretic peptide (Pro-BNP) circulates in human plasma. Biochem Biophys Res Commun 214:1175-1183, 1995

32. Martinez-Rumayor A, Richards AM, Burnett $\mathrm{JC}$, et al: Biology of the natriuretic peptides. Am J Cardiol 101:3-8, 2008

33. Wang TJ, Larson MG, Levy D, et al: Plasma natriuretic peptide levels and the risk of cardiovascular events and death. N Engl J Med 350:655-663, 2004

34. Black S, Kushner I, Samols D: C-reactive protein. J Biol Chem 279:48487-48490, 2004

35. Singh SK, Suresh MV, Voleti B, et al: The connection between C-reactive protein and atherosclerosis. Ann Med 40:110-120, 2007

36. Shishehbor MH, Bhatt DL, Topol EJ: Using C-reactive protein to assess cardiovascular disease risk. Cleve Clin J Med 70:634-640, 2003

37. Todd J, Freese B, Lu A, et al: Ultrasensitive flow-based immunoassays using single-molecule counting. Clin Chem 53:1990-1995, 2007

38. Sluysmans T, Colan SD: Theoretical and empirical derivation of cardiovascular allometric relationships in children. J Appl Physiol 99:445-457, 2005

39. Lipsitz SR, Fitzmaurice GM, Ibrahim JG, et al: Parameter estimation in longitudinal studies with outcome-dependent follow-up. Biometrics 58:621630,2002

40. Fitzmaurice GM, Lipsitz SR, Ibrahim JG, et al: Estimation in regression models for longitudinal binary data with outcome-dependent follow-up. Biostatistics 7:469-485, 2006

41. Lipshultz SE: Ventricular dysfunction clinical research in infants, children and adolescents. Prog Pediatr Cardiol 12:1-28, 2000

42. Ratnasamy $C$, Kinnamon DD, Lipshultz SE, et al: Associations between neurohormonal and inflammatory activation and heart failure in children. Am Heart J 155:527-533, 2008

\section{Affiliations}

Steven E. Lipshultz, Tracie L. Miller, Rebecca E. Scully, and Jacqueline M. Henkel, University of Miami Miller School of Medicine; Steven E. Lipshultz and Tracie L. Miller, University of Miami Sylvester Comprehensive Cancer Center, Miami, FL; Stuart R. Lipsitz, Brigham and Women's Hospital; Nader Rifai, Harvard Medical School; Lewis B. Silverman, Donna S. Neuberg, Suzanne E. Dahlberg, and Stephen E. Sallan, Dana-Farber Cancer Institute, Harvard Medical School; Lewis B. Silverman and Stephen E. Sallan, Children's Hospital Boston; Steven D. Colan, Children's Hospital Boston and Harvard Medical School, Boston, MA; Barbara L. Asselin, University of Rochester School of Medicine and Dentistry, Rochester, NY; Uma H. Athale, McMaster University, Hamilton, Ontario; Caroline Laverdière, Sainte Justine Hospital, Montreal; Bruno Michon, Le Centre Hospitalier de L'Universite, Laval, Quebec, Canada; Luis A. Clavell, San Jorge Children's Hospital, Puerto Rico; and Marshall A. Schorin, Inova Fairfax Hospital for Children, Fairfax, VA. 